\title{
Correcting for finite probe diameter in the dual probe heat pulse method of measuring soil water content
}

\author{
$\underline{\text { J.H. Knight }}^{\text {a }}$ and G.J. Kluitenberg ${ }^{\text {b }}$ \\ ${ }^{a}$ Department of Environmental Sciences, The University of Sydney, Sydney, NSW. \\ ${ }^{b}$ Department of Agronomy, Kansas State University, Manhattan, Kansas, USA. \\ Email:john.knight@sydney.edu.au
}

\begin{abstract}
This paper provides a simple correction to the measurement of soil volumetric heat capacity (and soil water content) using a dual probe heat pulse device with instantaneous heating, to allow for rods of finite radius and heat capacity. The correction is based on the spatial weighting function of Knight et al. (2007) and checked using the identical cylinders perfect conductor (ICPC) theory of Knight et al. (2012).
\end{abstract}

The dual probe heat pulse (DPHP) method uses two parallel rods to estimate soil volumetric heat capacity and hence volumetric water content. Most of the heat capacity of wet soil is due to its water content, and so the water content has a known relation to the heat capacity. A known pulse of heat applied to the emitter rod causes the temperature at the receiver rod to rise and then fall. The time and the size of the maximum temperature rise at the receiver are measured. Assuming that the thermal properties of the soil are spatially uniform, that the heat pulse is applied instantaneously, and that the rods have zero diameter and zero thermal capacity, the standard solution of the forward problem gives a formula for the maximum temperature rise in terms of the thermal capacity of the soil and the known parameters of the problem. Campbell et al. (1991) inverted this to give a solution of the inverse problem as a simple explicit formula for the soil heat capacity in terms of the maximum temperature rise and the known probe parameters.

Kluitenberg et al. (1993) and Bristow et al. (1994) introduced a calculation method that accounts for the finite probe heating duration, but requires the evaluation of the exponential integral function. Knight and Kluitenberg (2004) derived a simple power series approximation which converges well as long as the heating duration is not too big compared to the time to maximum temperature. This method is simple enough to be implemented on a small computer chip in a device. However it has been observed that the measurements are less accurate when the soil is dry, and this is likely to be due to the finite radius and finite heat capacity of the rods being neglected in the existing analysis. The semi-analytical solution of Knight et al. (2012) assumes that each rod is a perfect conductor, and that the presence of the receiver does not affect the temperature field near the emitter. They derived a solution of the forward problem in the Laplace transform domain and inverted it numerically, finding that the probe radius and heat capacity have a significant effect on the time and magnitude of the maximum temperature at the receiver, and hence on the accuracy of the method.

The spatial weighting function theory of Knight et al. (2007) for probes of zero radius provides an approximate method of calculating the apparent measured heat capacity of the soil-probe system as a weighted average of the heat capacity of the rods and the heat capacity of the surrounding soil, with the weights calculated by numerical integration and dependent on the probe radii. The weights need only be calculated once for a given probe geometry. This formula can easily be inverted to give a simple correction procedure which gives the soil thermal capacity from the measured apparent thermal capacity of the probesoil mixture and the known probe heat capacity. As expected, a larger correction is necessary for dry soil than for wet soil, and the correction is zero when the soil and probe heat capacities happen to be the same. The procedure is simple enough to be implemented on a computer chip with limited memory and processing capacity.

The accuracy of the procedure was checked by solving the forward problem of calculating the maximum temperature rise with known soil heat capacity and probe parameters using the semi-analytical solution of Knight et al. (2012). An iteration procedure was then used for the inverse problem to calculate the soil heat capacity given the measured temperature rise. The agreement with the approximate value given by the spatial weighting procedure is extremely good.

It is relatively straightforward to extend the theory given here for instantaneous heating to the case of finite heating duration.

Keywords: Soil water measurement, Dual probe heat pulse (DPHP), finite probe diameter 


\section{INTRODUCTION}

The dual-probe heat-pulse (DPHP) method of measuring soil volumetric heat capacity and hence water content was developed by Campbell et al. (1991). They used two parallel probes, each a long rod of radius $a$ small compared to their spacing $L$. Figure 1 shows a DPHP device used by Heitman et al. (2003). Campbell et al. (1991) assumed that a heating pulse of $Q \mathrm{~J} \mathrm{~m}^{-1}$ was applied instantaneously to the heating rod of zero radius and heat capacity, and that this caused the temperature to rise at the receiver rod, also of zero thickness and heat capacity. If $C_{s} \mathrm{~J} \mathrm{~m}^{-3} \mathrm{~K}^{-1}$ and $\lambda \mathrm{W}$ $\mathrm{m}^{-1} \quad \mathrm{~K}^{-1}$ are the heat capacity and thermal conductivity of the soil, with $\kappa=\lambda / C_{s} \mathrm{~m}^{2} \mathrm{~s}^{-1}$ the thermal diffusivity, then the temperature rise at the receiver at time $t$ is (Carslaw and Jaeger, 1959)

$$
T(L, t)=\frac{Q}{4 \pi \lambda t} \exp \left(-\frac{L^{2}}{4 \kappa t}\right) .
$$

The temperature rise has a maximum $T_{\mathrm{m}}$ at time $t_{m}=\frac{L^{2}}{4 \kappa}$. Campbell et al. (1991) inverted this to get a measured estimate $C_{\mathrm{m}}$ for $C_{s}$ as

$$
C_{m}=\frac{Q}{e \pi L^{2} T_{m}}
$$

which has the virtue of simplicity.

In practice the heating is not instantaneous but is

Figure 1. Configuration of a DPHP device.
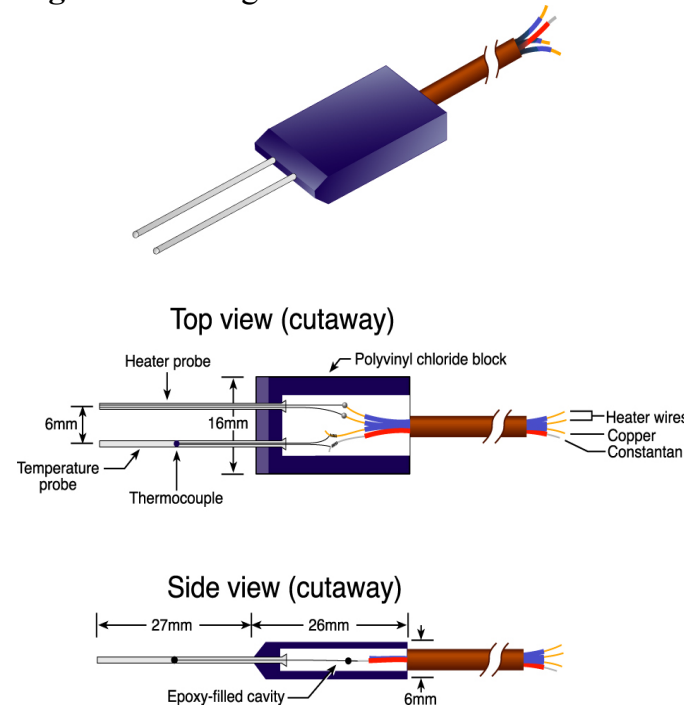

applied over a finite time interval, with $8 \mathrm{~s}$ being typical. Kluitenberg et al. (1993) and Bristow et al. (1994) introduced a calculation method that accounts for the finite probe heating duration, but requires the evaluation of the exponential integral function which is not routinely available on computers. Knight and Kluitenberg (2004) derived a simple power series approximation which converges well as long as the heating duration is not too large compared to the time to maximum temperature. This method is simple enough to be implemented in a spreadsheet, or on a small computer chip in a device.

Even when the finite heating duration is taken into account, direct comparisons for materials of known thermal properties have found that the estimates of $C_{S}$ are not as accurate as expected, especially when the soil is dry. The errors can be reduced to some extent by using a calibrated "apparent" spacing $L$ instead of the real physical spacing. This is likely to be due to the finite radius and finite heat capacity of the rods being neglected in the existing analysis. There has been a tendency to make the probes thin to conform with the theory, but this means that they are more likely to bend in hard soils. An analysis which took into account probe thickness would encourage the design of more rigid probes.

\section{THE SEMI-ANALYTICAL SOLUTION}

To test the hypothesis that the finite radius and heat capacity of the probes are important, Knight et al. (2012) derived a semi-analytical solution for probes of finite radius and heat capacity. They assumed that each rod is a perfect conductor, and that the presence of the receiver does not affect the temperature field near the emitter. Under these assumptions they used a Bessel function addition theorem to derive a solution in the Laplace transform domain of the forward problem for the temperature of the receiver given the soil heat capacity $C_{s}$. If the temperature of the receiver as a function of time is $V(t)$, then its Laplace transform as a function of the Laplace variable $p$ is defined as $\widehat{V}(p)$ with

$$
\widehat{V}(p)=\int_{0}^{\infty} V(t) e^{-p t} d t .
$$

Knight et al. (2012) found that the transform of the receiver temperature for instantaneous heating is

$$
\widehat{V}(p)=\frac{Q K_{0}(\mu L)}{2 \pi \lambda} \hat{v}^{2}
$$

with $K_{n}(z)$ the modified Bessel function of the second kind of order $n$ and argument $z, \mu=\sqrt{p / \kappa}$, and the transfer function for each probe 
Knight and Kluitenberg, Correcting for finite probe diameter in the dual probe heat pulse method

$$
\hat{v}(p, a, \beta)=\frac{1}{\mu a\left[K_{1}(\mu a)+(\mu a \beta / 2) K_{0}(\mu a)\right]},
$$

with $\beta=C_{0} / C_{s}$ the ratio of the probe heat capacity $C_{0}$ and the soil heat capacity. When the probe radius $a$ is very small the transfer function is close to unity and the Laplace transform of the temperature is

$$
\hat{V}(p)=\frac{Q K_{0}(\mu L)}{2 \pi \lambda}
$$

corresponding to the temperature given by (1), as in Carslaw and Jaeger (1959). Knight et al. (2012) inverted (4) numerically using the Stehfest (1970) method to find the temperature rise in the time domain. They found that the probe radius and heat capacity have a significant effect on the time and magnitude of the maximum temperature at the receiver, and hence on the accuracy of the method. Figure 2 shows the time course of the temperature rise at the receiver for three different dimensionless probe radii $\alpha, \alpha=a / L$, of $0.01,0.1$ and 0.2 , and the ratio of heat capacities of $\beta=2$, with soil thermal conductivity $\lambda=0.5 \mathrm{~W} \mathrm{~m}^{-1} \mathrm{~K}^{-1}$ and heat capacity $C_{s}=2 \times 10^{6} \mathrm{~J} \mathrm{~m}^{-3} \mathrm{~K}^{-1}$. The clear differences in the maximum temperature, and also in the time to the maximum, are evident for the different dimensionless probe radii.

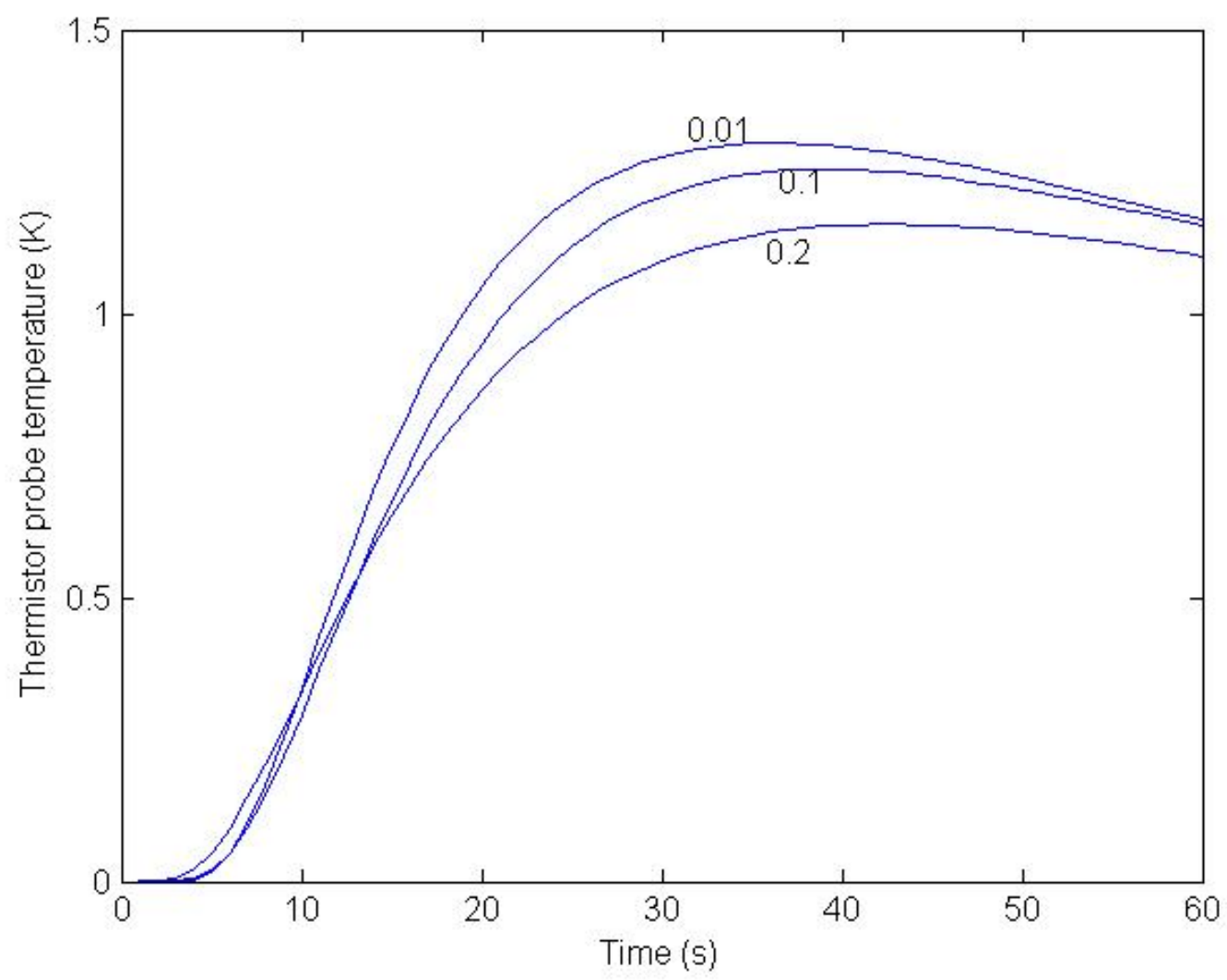

Figure 2. Temperature rise with time at the receiver probe, for the three dimensionless probe radii values $\alpha$ of $0.01,0.1$ and 0.2 , and for $\beta=2$.

\section{THE SPATIAL WEIGHTING FUNCTION}

Knight et al. (2007) derived the spatial weighting function which gives the relative weighting that the measurement method gives to values of the soil heat capacity $C_{S}$ at various positions around the probes. For small variations around the probes we have that

$$
C_{m}=\int_{-\infty}^{\infty} \int_{-\infty}^{\infty} C_{s}(X, Y) W(X, Y) d X d Y
$$

with the normalized weighting function having the property that 
Knight and Kluitenberg, Correcting for finite probe diameter in the dual probe heat pulse method

$$
\int_{-\infty}^{\infty} \int_{-\infty}^{\infty} W(X, Y) d X d Y=1 .
$$

For the DPHP instrument with rods of zero thickness and instantaneous heating the normalized weighting function has the form as a function of dimensionless space variables $X$ and $Y$, with $X=x / L, Y=y / L$

$$
W(X, Y)=\frac{2}{\pi}\left[\left(2 R^{2}-\frac{1}{2}\right) K_{0}\left(2 R_{1} R_{2}\right)+2 R_{1} R_{2} K_{1}\left(2 R_{1} R_{2}\right)\right] \exp \left(-2 R^{2}+\frac{1}{2}\right)
$$

The emitter is at position $(-1 / 2,0)$ and the receiver is at $(1 / 2,0)$. The distances $R_{1}$ and $R_{2}$ are the dimensionless distances from $(-1 / 2,0)$ and $(1 / 2,0)$ respectively, and $R$ is the dimensionless distance from the origin. Taking the limit as $R \rightarrow \frac{1}{2}, R_{1} \rightarrow 1, R_{2} \rightarrow 0$ gives the values at the centres of the probes,

$$
W\left( \pm \frac{1}{2}, 0\right)=\frac{2}{\pi}
$$

Figure 3 is a surface plot of the normalized weighting function (9) showing the positions of the two rods of zero thickness.

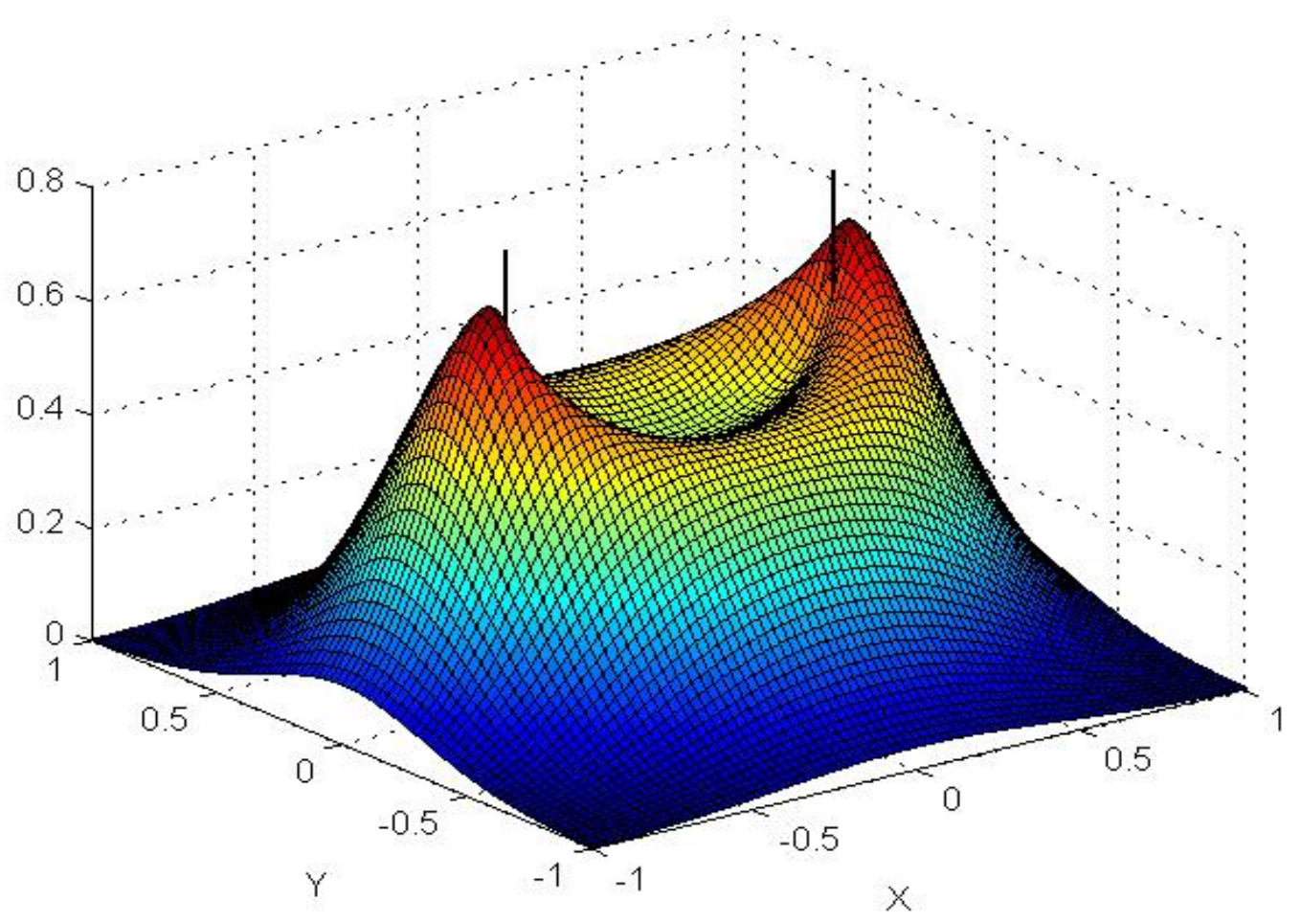

Figure 3. Surface plot of (9) showing the positions of the two rods of zero thickness.

For the case with the soil around the probes having a uniform heat capacity $C_{S}$ and the probe material having an assumed uniform heat capacity $C_{0}$, the integral (8) takes the simple form

$$
C_{m}=C_{0} w_{0}+C_{s}\left(1-w_{0}\right)
$$

with the coefficient $w_{0}$ being the integral of the normalized weighting function (9) over the crosssection of both probe areas, 
Knight and Kluitenberg, Correcting for finite probe diameter in the dual probe heat pulse method

$$
w_{0}(\propto)=2 \int_{0}^{\propto} \int_{0}^{2 \pi} W(X, Y) R_{1} d \varphi d R_{1}
$$

where $R_{1}$ is the local distance from the probe rod axis and $\varphi$ is the angle around the axis. For small dimensionless probe radius $\propto$ we can assume that the average value of $W$ is close to its value at the probe centre, and so

$$
w_{0}(\propto) \sim 4 \propto^{2}
$$

The integration (11) was done numerically for various values of $\propto$ and the ratio of $w_{0}(\propto) / \propto^{2}$ plotted in Figure 4. The ratio is 4 for zero probe thickness and is approximately 3.85 for a probe with $\propto=0.1$.

\section{CORRECTION FOR FINITE PROBE RADIUS}

The formula (10) can easily be rearranged to give an approximate solution to the inverse problem,

Figure 4. Dependence of weight $w_{0}$ on radius of probe

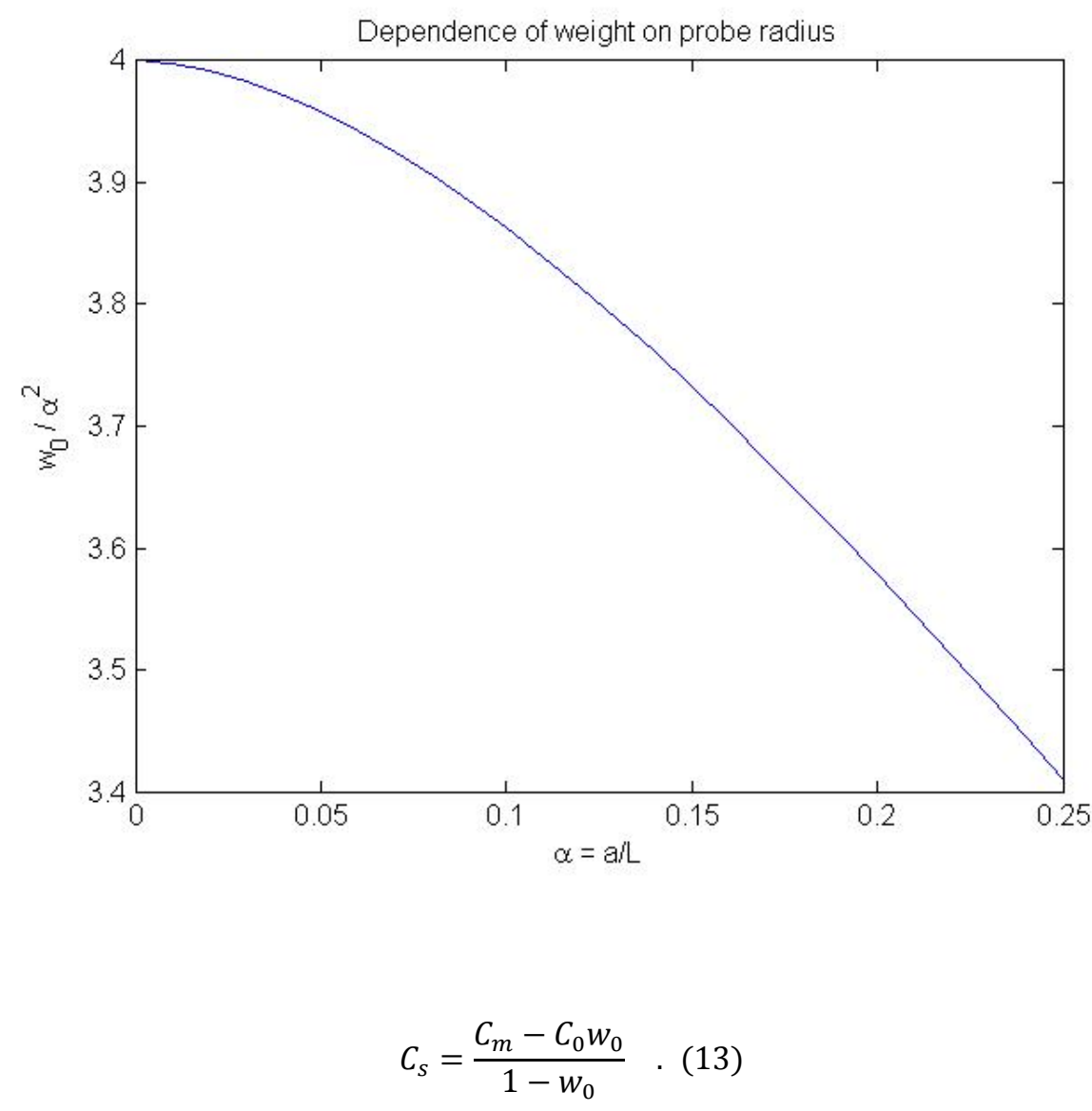

For the case of instantaneous heating, the weight $w_{0}$ needs to be calculated only once initially for a given probe configuration, and then (13) gives a simple correction to the apparent measured values of soil thermal capacity. The procedure can be applied retrospectively to measured values, as long as the probe parameters are known.

\section{CHECKING AGAINST ICPC CALCULATIONS}

For a typical probe configuration of Basinger et al. (2003) the predictions of (13) were checked against the semi-analytical solution of Knight et al. (2012), used in inverse mode. With $\alpha=0.1, C_{0}=2.84$, numerical integration gave $w_{0}=3.85 \propto^{2}$. A simple iteration procedure was then used for the inverse problem to 
calculate the soil heat capacity given a measured temperature rise $T_{m}$ with values from 0.5 to $2 \mathrm{~K}$. Figure 5 shows the results. Results are shown for the percentage corrections made with the semi-analytical model, and with (13) for the calculated value $w_{0}=3.85 \propto^{2}$, and also with the assumed value $w_{0}=4 \propto^{2}$ corresponding to a thin rod. The results of the three correction methods are surprisingly close. As expected, the greatest corrections are necessary when $T_{m}$ is largest, corresponding to a dry soil with low thermal capacity. Using the calculated value of $w_{0}$ gives a better correction than just using $w_{0}=4 \propto^{2}$, but the difference is not great.

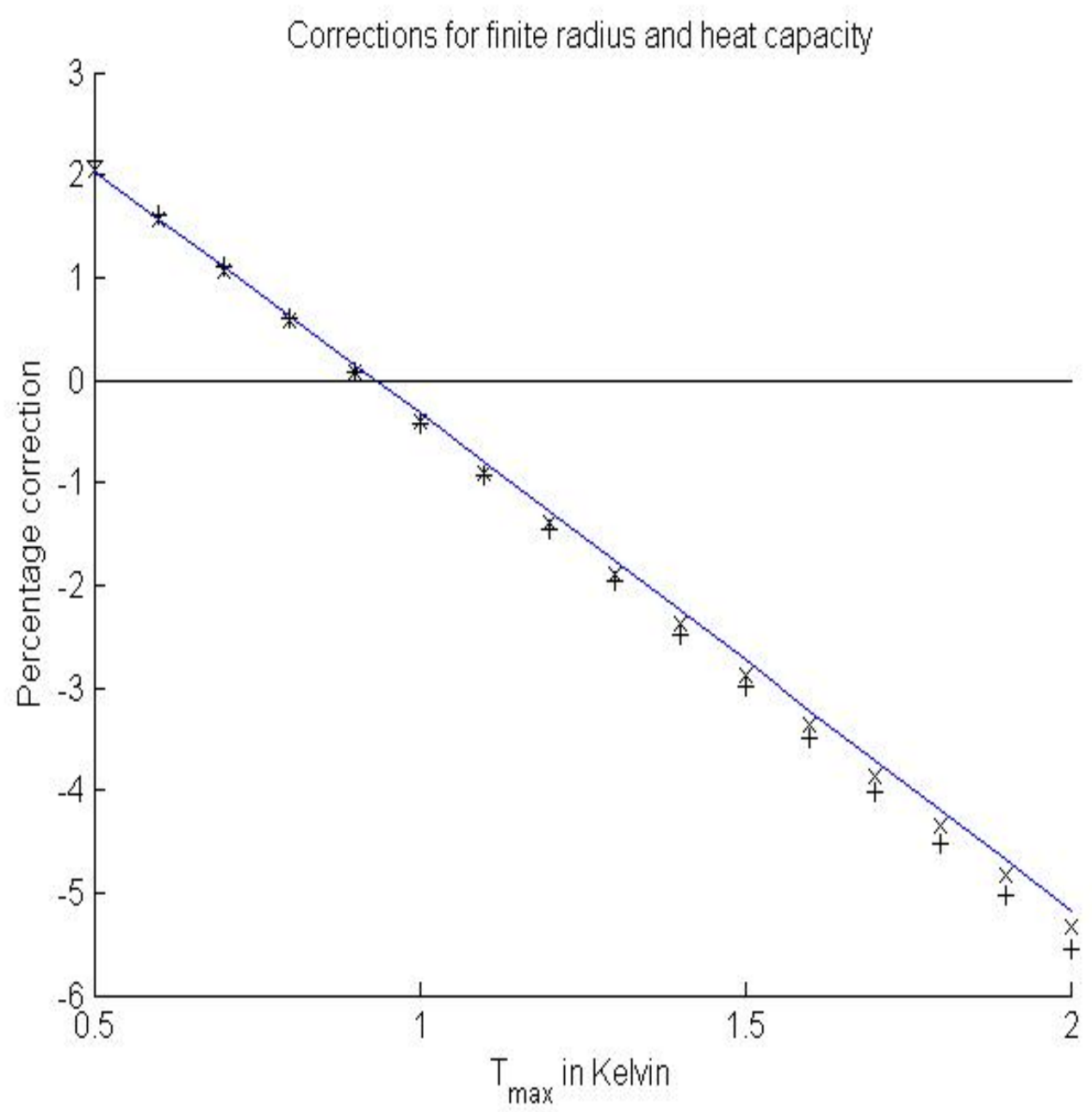

Figure 5. Percentage corrections for various estimates of soil heat capacity. The measured value with no correction is the horizontal line. The solid line is the correction calculated by the semi-analytical solution in inverse mode. The points $(\mathrm{x})$ show the percentage correction from (13) with the calculated value $w_{0}=3.85 \propto^{2}$, and the crosses $(+)$ show the same expression (13) with the assumed value $w_{0}=4 \propto^{2}$.

\section{DISCUSSION AND CONCLUSIONS}

A simple correction formula has been derived to correct measurements of soil heat capacity using the DPHP method with instantaneous heating for the finite radius and finite heat capacity of the probes. It is very much simpler than using the semi-analytical solution of Knight et al. (2012) in inverse mode to infer soil thermal capacity from measurements of maximum temperature rise, and the results are almost as good.

It is relatively straightforward to extend the theory given here for instantaneous heating to the case of finite heating duration. 
Knight and Kluitenberg, Correcting for finite probe diameter in the dual probe heat pulse method

\section{ACKNOWLEDGMENTS}

JHK thanks the Department of Agronomy and Kansas State University generally for hospitality while some of this work was done.

\section{REFERENCES}

Campbell, G.S., Calissendorff, C., and Williams, J.H. (1991). Probe for measuring soil specific heat using a heat-pulse method. Soil Science Society of America Journal, 55, 291-293.

Carslaw, H.S., and Jaeger, J.C. (1959). Conduction of heat in solids. Clarendon Press, Oxford. Second Edition.

Basinger, J.M., Kluitenberg, G.J., Ham, J.M., Frank, J.M., Barnes, P.L., and Kirkham, M.B. (2003). Laboratory evaluation of the dual-probe heat-pulse method for measuring soil water content. Vadose Zone Journal, 2, 389-399.

Bristow, K.L., Kluitenberg, G.J., and Horton, R. (1994). Measurement of soil thermal properties with a dualprobe heat-pulse technique. Soil Science Society of America Journal, 58, 1288-1294.

Heitman, J.L, Basinger, J.M., Kluitenberg, G.J., Ham, J.M., Frank, J.M., and Barnes, P.L. (2003) Field evaluation of the dual-probe heat-pulse method for measuring soil water content. Vadose Zone Journal, 2, 552-560.

Kluitenberg, G.J., Ham, J.M., and Bristow, K.L. (1993). Error analysis of the heat-pulse method for measuring soil volumetric heat capacity. Soil Science Society of America Journal, 57, 1444-1451.

Knight, J.H., Jin, W., and Kluitenberg, G.J. (2007). Sensitivity of the dual-probe heat-pulse method to spatial variations in heat capacity. Vadose Zone Journal, 6(4), 746-758.

Knight, J.H., and Kluitenberg, G.J. (2004). Simplified computational approach for dual-probe heat-pulse method. Soil Science Society of America Journal, 68(2), 447-449.

Knight, J.H., Kluitenberg, G.J., Kamai, T., and Hopmans, J.W. (2012). Semianalytical solution for dualprobe heat-pulse applications that accounts for probe radius and heat capacity. Vadose Zone Journal, doi:10.2136/vzj2011.0112

Stehfest, H. (1970). Algorithm 368: Numerical inversion of Laplace transforms. Communications of the Association for Computing Machinery, 13, 47-49. 